\title{
Robot-assisted sleeve gastrectomy in patients with obesity with a novel Chinese domestic MicroHand S】 surgical system
}

\author{
Weizheng Li \\ Central South University Third Xiangya Hospital \\ Kang Kong \\ Tianjin University \\ Pengzhou Li \\ Central South University Third Xiangya Hospital \\ Guohui Wang \\ Central South University Third Xiangya Hospital \\ Beibei Cui \\ Central South University Third Xiangya Hospital \\ Liyong Zhu \\ Central South University Third Xiangya Hospital \\ Shaihong Zhu ( $\nabla$ shaihongzhu@126.com ) \\ Central South University Third Xiangya Hospital
}

\section{Research article}

Keywords: Robot-assisted surgery, Sleeve gastrectomy, Obesity

Posted Date: November 6th, 2020

DOI: https://doi.org/10.21203/rs.3.rs-56373/v2

License: (c) (i) This work is licensed under a Creative Commons Attribution 4.0 International License. Read Full License 


\section{Abstract}

Background: A new member has been added to the Chinese MicroHand surgical robot family, developed based on the successful application of control algorithms. As a benefit of using these specialized control algorithms, the motion mapping relation can be built up without the help of a built-in image system, resulting in a novel Chinese domestic surgical robot with two arms called MicroHand S\&, which is different from the former MicroHand S and da Vinci systems. The analysis technique of clinical practise using the Chinese domestic surgical robot to perform

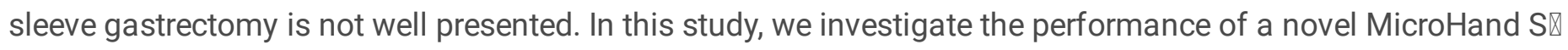
robotic platform in patients with obesity.

Methods: From March 2018 to April 2019, a total of 7 patients affected by obesity were operated on with the robotassisted technique using the MicroHand $\mathrm{S} \otimes$ surgical system. Data regarding demography, surgical procedure and the 3-month outcome postoperation were collected.

Results: There were 2 female and 5 male patients with a mean age of $34.0 \pm 11.4$ years. The mean operative time was $166.4 \pm 16.1$ minutes. None were converted to open surgery. All anthropometry indices improved significantly at 3 months postoperatively.

Conclusions: In conclusion, the Chinese domestic robot surgical system MicroHand $\mathrm{\otimes}$ could be feasibly and safely applied in sleeve gastrectomy in patients with obesity. This robotic platform has presented its own obvious advantages.

\section{Background}

Obesity has growing prevalence worldwide, causing a series of severe problems in public health [1]. Compared to non-surgical interventions, bariatric surgery can dramatically reduce body weight in a lasting manner and ameliorate comorbidities in patients with obesity $[2,3]$. At present, hundreds of thousands of patients with obesity receive bariatric surgery every year [4]. Laparoscopic sleeve gastrectomy (LSG) has become the most common procedure in bariatric surgery according to an estimation of the International Federation for the Surgery of Obesity and Metabolic Disorders (IFSO) [5]. However, it is difficult to dissociate and release the stomach fundus limited by the laparoscopic technique applied to anatomically deep stomach fundus, especially for patients with obesity.

With the development of robotic surgical systems, robotic surgery has appeared as a new category of minimally invasive surgery [6]. Robotic technology with multiple degrees of freedom (DoF) and tridimensional (3D) imaging can improve operating dexterity, locating precision, and hand-eye coordination in surgery. The robot surgical system compensates for the technical limitations of laparoscopic instruments and solves many problems caused by human restrictions, such as fatigue and low precision.

The robotic platform has gained widespread utilization in bariatric surgery for the treatment of morbid obesity in recent years [7]. The Metabolic and Bariatric Surgery Accreditation and Quality Improvement Program (MBSAQIP) database showed that, in the last few years, the da Vinci robotic platform has increasingly emerged as an attractive technology in bariatric surgery $[8,9]$. At present, da Vinci sleeve gastrectomy has been carried out, showing good performance $[10,11]$. Therefore, robot-assisted sleeve gastrectomy is a new form of surgery to bring a new experience to patients with obesity, especially for surgeons operating on patients with obesity.

However, the high costs of the da Vinci robotic system make it very difficult to routinely apply it to bariatric surgery $[11,12]$. Moreover, its expensive annual maintenance requirements and surgical consumables limit the application of 
the da Vinci surgical system in China. However, recently, the Chinese domestic MicroHand surgical system was developed with a compact structure and low cost. Similar to the da Vinci surgical robot, the MicroHand series surgical robot is a kind of master-slave robot [13].

Recently, the MicroHand surgical system added a novel robot called the MicroHand S\ Chinese domestic surgical robot. The MicroHand S $\triangle$ system was developed in 2015 by two universities. We have witnessed the performance of the MicroHand S robotic platform developed in 2013 in primary clinical applications. According to previous studies $[14,15]$, MicroHand S has been applied to appendectomy, cholecystectomy, and right hemicolectomy, without deep organs involved. Therefore, we are highly interested in a novel two-arm robot used in deep organs. The purpose of this study is to investigate the performance of the MicroHand $\mathrm{S} \otimes$ robotic platform by accomplishing the surgical task analysis of sleeve gastrectomy in patients with obesity.

\section{Methods}

Seven patients with obesity who met the metabolic surgery criteria set by the Chinese Society for Metabolic \&

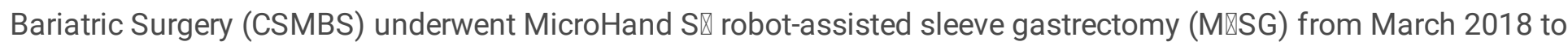
April 2019. All patients suffered from fatty liver with hypertrophic hepatic lobes. Perioperative overall operative time, preoperative setup time, intraoperative blood loss, hospital stays, and perioperative complications were recorded. Anthropometry data, including body weight, body mass index (BMI) and waist circumference (WC) preoperatively and 3 months postoperatively were collected. Percentage excess weight loss (\% EWL) was estimated in the following 3 months. $\% \mathrm{EWL}=$ (weight loss/baseline excess weight) $\times 100 \%$, where baseline excess weight $=$ baseline weight ideal weight. The ideal weight is based on a person's weight at a BMI of $25 \mathrm{~kg} / \mathrm{m}^{2}$. All results were expressed as a mean \pm standard deviation. The paired-samples $T$ test was used, and the significance level was set at a two-tailed $a=0.05$. The surgeons had sufficient training to adapt well to the MicroHand $\mathrm{S} \otimes$ robot. Written informed consent was obtained from each patient. This study was authorized by the ethics committee of the hospital and registered in the National Institutes of Health website: www.clincaltrials.gov. The registration identifier is NCT02752698.

\section{Overview of the MicroHand SI robot surgical system}

The MicroHand S $\nabla$ robot surgical system mainly consists of a surgeon console, a slave robot and a video vehicle (Fig. 1), the structure and appearance of which are distinctly different from those of the da Vinci surgical system. The preloaded cable-driven system and harmonic reducers make it light in weight, compact in structure, and enable a large range of motion. Compared to the MicroHand $\mathrm{S}$, this robot was upgraded successfully in configuration, such as being equipped with an ultrasonic scalpel, a master-slave motion scaling function and an audible alarm function. Both the scaling and alarm functions are integrated into the control panel for quick manual manipulation. Benefitting from using specialized control algorithms, the motion mapping relation can be built up without the help of a built-in image system, which are different from the MicroHand S and da Vinci systems. Therefore, the two-arm MicroHand $S \otimes$ is compatible with conventional endoscopic image systems in hospitals, in which the manipulator used to hold the endoscope is abolished. Thanks to this design, the cost, volume and weight of the MicroHand S $\otimes$ can be further reduced.

The surgeon console allows the surgeon to control multi-DoF instruments by operating master manipulators and foot pedals. The two master manipulators conform to the ergonomic engineering facilitating to filter out hand tremors, relieve hand fatigue and improve precision. Owing to the embedded controller, the motions input by operating master manipulators outside the abdomen can be mapped to the end-effector motions of instruments inside the abdomen cavity exactly. The reproduced motions of the instruments following the master manipulators 
are activated by grasping the button on the handle. Then, incremental motion is used to reposition the master manipulators during surgery to solve the mutual interference or motion limits of the master manipulators, which is implemented by the clutch mechanism fired by pinching the clamp to disengage the instrument motion from the corresponding motion of the master manipulators. The control panel is used for system initialization and to establish the initial values of certain key parameters prior to surgery. The motion scale between the master and the slave is adjustable among different proportions, such as $3: 1,6: 1$ and $10: 1$, by simply clicking the keys on the control panel at any time during the entire procedure (as shown in Fig. 2).

This master-slave motion scaling function improves the accuracy by switching the motion scale during the operation. A 3:1 proportional motion control means the movement of the slave manipulator takes one-third of the master manipulator movement. For example, when dissociating the gastrocolic ligament using the ultrasonic scalpel, the surgeon usually chooses 3:1 to complete resection quickly. When isolating the short gastric vessel, the surgeon can choose 10:1 to refine the operation. The master-slave motion mapping strategies of MicroHand S $\nabla$ are a unique design. The setting can be turned up in fine operations and turned down in extensive operations. This design can improve the safety and speed up the process of the operation and has been well applied in operations.

The audible alarm function is attributed to the sensor equipped in each passive joint, which is to guarantee operation safety. The control system compares the angular positions provided by the sensors and those obtained through the kinematic calculation at each controller time-step. Once the error between them exceeds a certain range, the robot is stopped immediately, and an alarm sounds in the control panel. The surgeon needs clear out the fault first by clicking the button on the control panel and then continue the surgical procedure. The imaging system transmits a stable 3D view in an open-field way (Fig. 3a). Benefitting from the open high-definition 3D view of MicroHand S区, an easier real-time discussion and teaching intraoperative is allowed for, which is inconvenient with a closed image viewer integrated in the console in da Vinci (Fig. 3b). In addition, it is helpful to relieve the surgeon's neck fatigue without persistently laying the head down against the image viewer for a long time.

The operating arm system is optimized with two movable manipulators installed on the swivel head used to place the instruments (Fig. 4a). The structure of the instrument is designed optimally on the basis of the kinematic analysis together with the ergonomic index. To ensure that the surgical robot can perform the suture motion, which is largely a rotation about the bisector line of the two jaws of the instrument, an end rotational motion needs to be realized. Based on this, a separated roll joint is designed at the distal end of the instrument (Fig. 4b). Eventually, the system allows the manipulator to move with seven DoFs beyond the laparoscopic surgery, technically.

\section{The technical advantages in patient-robot interaction}

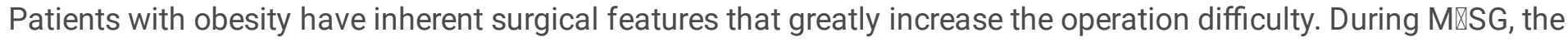
target organ is the stomach in the upper abdominal region; the robot is used to assist in operations. In terms of the LSG surgical requirements, problems related to thick abdominal walls, a mass of visceral fat, deep stomach fundus and limited space to manoeuvre the instrument are always encountered. At the same time, the anterior and posterior diameters of the abdominal cavity are large, and stomach fundus and part of the gastric body in patients with obesity are covered by the plump left liver lobe. However, MicroHand $\mathrm{S} \otimes$ has some particular features, especially in patient-robot interaction, to overcome the difficulties in exposure and operation.

It is convenient to locate the slave robot owing to its crossbeam design and flexible swivel head. The route of movement up and down the column of the slave robot is long-range, which is enough to overcome the elevated bed after patients with obesity lay on the bed. The surgeon need not intentionally turn down the bed to adapt to the slave 
robot. This design can free up space around the patient's head, which makes general anaesthesia easier during a robotic surgery. It is beneficial to place and settle surgical equipment, including the anaesthetist cabinet, several sterile trays, display monitors, several instrument cupboards, and so on, to satisfy operation room area requirements. However, many pieces of equipment are always stored in every area until needed for surgery. This compact robot does not occupy much room around the patient, keeping the surgeon or assistants close to the patient.

During the whole intervention of the $\mathrm{M} \otimes \mathrm{SG}$, benefitting from its optimal design, although patients with obesity are large, we can always place the slave robot acceptably without repeated adjustments. The slave robot of the MicroHand $\mathrm{S} \otimes$ is well-suited to this situation and can be placed without the need to move and dock the robotic cart several times. It is not affected by the position of the operating bed only meeting the basic placement principle, which is simply that the slave robot is near to the lesion side. The operation site can be completely covered by the workspace of the robot to avoid the need to reposition the robot during the surgery. In the da Vinci surgical system, the slave robot must be placed at a special site near the operation bed. Therefore, the position of the surgical cart is simpler than in the da Vinci system, without repositioning during the entire procedure.

The two manipulators of the slave cart have long arms, which are suited for long-scale adjustments. The kinematic design of the robot arm can fulfil the incision point constraint [16]. The number of joints is set reasonably, and the length proportion of each arm is set appropriately. In the contracted state, each arm is folded together, occupying little space. In the open state, each arm is fully and freely extended to generate a very wide operating field for encircling, avoiding external collisions of the arms. These designs not only meet the space requirements of the abdominal operating area but also meet the requirements of the operating triangle of the working instruments. Therefore, it is easy to position the surgical cart and trocars. The robot can release some of the space around the operating table that may be occupied by the robot arms compared with the da Vinci assisted system. The $450 \mathrm{~mm}$ length of the instruments, including robotic graspers, needle holder and ultrasonic scalpel, is longer than that of the da Vinci system, which can fulfil the motion requirements with a maximum moving range of $250 \mathrm{~mm}$ for the back and forth motion measured from the incision point.

The M $\forall S G$ port setup is more flexible than the LSG setup in spite of the incision point constraint. The DoFs of the laparoscopic rigid instruments are restricted to four, that is, three rotation motions and one translation motion by passing through fixed small incisions [17]. The mechanism of the robot arm part is composed of three active joints and three passive joints. A fixed point has been used to satisfy the incision point constraint by developing an optimized mechanism in the arm part [18]. The fixed point coincides in position with the location of the skin incision leading to positioning algorithms with roll angle and pitch angle, as shown in Fig. 5.

\section{Surgical technique: MDSG}

After general endotracheal anaesthesia was performed successfully, the patient was placed in the supine position with his or her legs by the side. After the abdomen was sterilely prepared and draped and a foley catheter was inserted, pneumoperitoneum was created with a Veress needle at a point $1 \mathrm{~cm}$ superior to the umbilical fossa up to a pressure of $14 \mathrm{mmHg}$. Then, a $12 \mathrm{~mm}$ trocar was inserted for placing a $45^{\circ}$ and 12-mm 3D laparoscope as the camera-holder port (A0). The distance between $\mathrm{A} 0$ and the umbilicus varied to achieve the proper angle of best visualization. The two ports for the robotic instrument arms (R1 and R2) were set up next. The distances for instrument port placement were measured after insufflation with individualized design, and at least a fist length (10 $\mathrm{cm}$ ) was maintained between all ports. R1 (8-mm MicroHand cannula) was established at the left midclavicular line superior to the A0 level. R2 (8-mm MicroHand cannula) was placed inside the right midclavicular line superior to the R1 level, with care taken to ensure that the route of the instrument was under the margin of the left liver. In particular, 
$\mathrm{R} 1$ was replaced by a 12-mm trocar as the camera-holder port during the stapling, provisionally. A0 was used as the stapler port, accordingly. In addition, another 5-mm port was placed at the anterior axillary line in the left hypochondrium as an assistant port (A1), which was used to retract the left lobe of the liver, assist stapling and so on.

The patient was placed in the steep reverse Trendelenburg position. The slave robot was brought from one of the available sites on the left side of the patient, and docking was performed in a short time. The camera-holder stood between the patient's legs. The assistant surgeon was on the left of the operating table. Although the fundus of the stomach was very close to the spine, which is far from the port site, especially for patients with obesity, the range of the instruments was sufficient to reach the fundus field and His angle to isolate tissue and to finish the separation of the fundus.

The two main steps of sleeve gastrectomy include the complete dissection and subsequent resection of the greater curvature and gastric fundus. Safe exposure and mobilization of the fundus are regarded especially as the crucial procedure of a sleeve gastrectomy. Gastrolysis was performed along the greater curvature of the stomach from the prepyloric region to the His angle using a MicroHand ultrasonic scalpel. The short gastric vessels from the gastrosplenic ligament and posterior gastric adhesions with the pancreas were divided. The left crus was completely defined so that the fundus was adequately mobilized. Instead of a bougie, a gastroscope was placed through the pylorus into the first part of the duodenum and was kept in place to guide the sleeve formation. The console surgeon applied traction on the stomach so that it was in the right orientation. Continuous stapling started $5 \mathrm{~cm}$ from the pylorus towards the His angle using a linear stapler through the $\mathrm{A} 0$ port to resect the stomach by a patient-side surgeon with adequate training in LSG skills. Instead, R1 was replaced by a 12-mm trocar with the laparoscope inserted. After gastric resection, gastroscopy with saline solution immersion was performed in all cases to rule out any leak, bleed, or obstruction. Finally, the resected stomach specimen was removed through the accessory port, and one drain was left in the peritoneal cavity. The port placement, operation room layout and detailed reality images of the robotic operating procedure are shown in Fig. 6.

\section{Results}

The patients with obesity included 2 females and 5 males. The mean age was $34.0 \pm 11.4$ years. The overall operating time was $166.4 \pm 16.1$ minutes, of which preoperative setup time was $27.5 \pm 2.5$ minutes. The mean hospital stay was $3.6 \pm 1.8$ days. Intraoperative blood loss was $20.8 \pm 3.6 \mathrm{ml}$. No serious complications occurred, such as margin leak, postoperative bleeding or conversion to open. Compared to the baseline, all anthropometry indices improved at 3 months postoperatively (see Table 1$)$. The percentage excess weight loss (\% EWL) at 3 months was $62.6 \pm 10.3$.

Table 1

Anthropometry indices at the baseline and 3 months post-M $₫ \mathrm{SG}$

\begin{tabular}{|c|c|c|}
\hline & Baseline $(n=7)$ & 3 months $(n=7)$ \\
\hline Weight (kg) & $106.3 \pm 19.1$ & $86.7 \pm 10.2^{*}$ \\
\hline $\operatorname{BMI}\left(\mathrm{kg} / \mathrm{m}^{2}\right)$ & $36.5 \pm 7.6$ & $29.7 \pm 4.0^{*}$ \\
\hline WC (cm) & $118.7 \pm 12.3$ & $103.3 \pm 11.8^{*}$ \\
\hline \%EWL & & $62.6 \pm 10.3$ \\
\hline \multicolumn{3}{|c|}{$\begin{array}{l}\text { MaSG- MicroHand S } ₫ \text { robot-assisted sleeve gastrectomy; BMI-body mass index; WC-waist circumference } \\
\% \text { EWL- percentage excess weight loss }\end{array}$} \\
\hline$* \mathrm{p}<0.05$ & & \\
\hline
\end{tabular}




\section{Discussion}

Owing to exciting anthropometric results, SG has currently become the commonest procedure in utilization of bariatric surgery. However, because SG has potential risk of promoting gastroesophageal reflux disease and Barrett esophagus, some procedures designed to prevent postoperative reflux have also gained a widespread diffusion [19, 20]. The clinical application experience to perform SG assisted by the MicroHand $\mathrm{S} \nabla$ is presented for the first time, but the risk of reflux after $M \otimes S G$ needs to be confirmed by a further large sample study.

In terms of SG surgical effectiveness results, all of the variables considered, such as BMI change and \%EWL, are similar to our laparoscopic data [21]. We introduced the course and have built a standardized technique by summing up our experience. The use of the MicroHand $\mathrm{S} \otimes$ robot during the entire intervention is smooth and comfortable. The operating surgeon is seated at the console; it is no longer necessary to force against the lever resistance of the abdominal wall or endure the discomfort of the body position. The tremor filtration obtains stable movement despite hand fatigue and minimizes the risk of secondary injury to healthy tissues.

In the da Vinci robot system, the preoperative setup procedure is cumbersome and time-consuming, and interference often occurs between the crowded robotic arms owing to large and heavy passive arms [22]. By comparing those manipulators, it can be observed that the robotic arms of the da Vinci surgical system are more complex and that more passive joints need to be adjusted before surgery compared with the MicroHand robot [23]. The design of the active slave manipulator combined with the passive arm and swivel head structure can fulfil the requirement of a fast preoperative setup procedure, as there is a quick-exchange interface designed at the end of each manipulator. The function is to realize the quick installation or removal of the surgical tools. Two arms with quick-exchange interface can save setup time. The total operation time is extended in M $\mathbb{S}$ G compared to LSG, by experience, mainly due to the preoperative setup procedure. However, it is far off set by the great advantages of robot-assisted technology especially in complex surgeries. Moreover, the preoperative setup time and the total operation time will be reduced reasonably with more M $\triangle \mathrm{SG}$ cases performed [24].

The MicroHand S $\nabla$ surgical robot is a master-slave robot, similar to the da Vinci surgical robot. However, this platform is different from the da Vinci robotic platform in many aspects of the original design. First, da Vinci surgical systems are equipped with four manipulators, including one laparoscope carrier and three working arms. MicroHand $\mathrm{S} \bigotimes$ systems are equipped with only two manipulators to perform surgical procedure and with an assistant holding the laparoscope, similar to conventional laparoscopic surgery. Second, instruments of the da Vinci surgical system are connected to the front end of the retractable manipulators, occupying too much space above the patient. Thus, the workspace reachable is reduced due to collisions between robot arms and other obstacles [22]. However, the two manipulators of MicroHand S $\varangle$ are suspended on the hoisting beam, thus occupying more upper useless space and reserving enough useful workspace for the assistant surgeon, effectively avoiding interference between instruments and surgeons. Third, the instruments in the da Vinci surgical system move in the roll-pitch-yaw way. However, the DoF arrangement of the instruments in MicroHand $\mathrm{S} \otimes$ take a roll-pitch-distal roll way. The DoF mechanism has diversity, as the configuration is different from the da Vinci system.

The two systems are different in many ways, such as the surgeon operation panel, manipulator design and imaging system. The two different systems complete surgery in different courses during surgical procedures with the respective robot techniques. The double-arm design is more conducive to the promotion of the MicroHand S $\otimes$ system in different endoscopic operations. The design of the MicroHand $\mathrm{S} \rrbracket$ arms requires the help of a hand holding the 
laparoscope, which is conducive to the wider range of the system to adapt to different parts of the endoscopic surgery at the present development stage. A new series design of the MicroHand system is proposed to meet the requirements of more complex operations. A prototype integrated with the slave manipulator carrying a laparoscope and/or a fourth one will be applied clinically soon.

In patients with obesity, the leverage resistance effect is obvious after the trocar passes through the thick abdominal

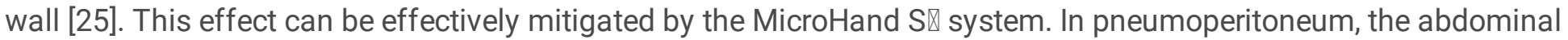
cavity is domed, and the abdominal wall is filled with gas in a uniform radian. Under the MicroHand S区 system, the setting of the joint module can further adjust the expanded position and state of the abdominal wall in the presence of pneumoperitoneum, which is conducive to intraoperative trocar regulation. Because the relative positions between the incision point and the slave manipulator are not so strictly determined in the manipulator with three passive joints, the robot can reach different operation positions by adjusting the height of the passive arm and by rotating the swivel head.

\section{Conclusions}

In conclusion, the Chinese novel surgical robot system MicroHand S $₫$ could be feasibly and safely applied in sleeve gastrectomy. The MicroHand $\mathrm{S} \otimes$ robotic platform has obvious advantages in routine use and will be applied widely to more complicated surgical procedures. At the same time, the medical cost of domestic robot-assisted surgery is expected to be reduced by more than half compared to that with the da Vinci robot. Therefore, more patients will benefit from a low-cost and easy-use surgical robot system with the commercialized MicroHand system in future. However, additional studies, including more clinical studies, should be carried out to verify the comprehensive performance of the developed surgical robot.

\section{Abbreviations}

LSG: Laparoscopic sleeve gastrectomy

IFSO: The International Federation for the Surgery of Obesity and Metabolic Disorders

DoF: Degrees of freedom

3D: Tridimensional

MBSAQIP: The Metabolic and Bariatric Surgery Accreditation and Quality Improvement Program

CSMBS: The Chinese Society for Metabolic \& Bariatric Surgery

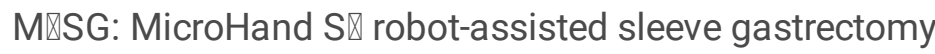

BMI: body mass index

WC: waist circumference

\% EWL: Percentage excess weight loss

\section{Declarations}




\section{Ethics approval and consent to participate}

This study was authorized by Institutional Review Board of the Third Xiangya Hospital, CSU. and registered in the National Institutes of Health website: www.clincaltrials.gov. The registration identifier is NCT02752698. Informed consent was obtained from all individual participants included in the study.

\section{Consent to publish}

Informed consent for publication was obtained from all individual participants included in the study.

\section{Availability of data and materials}

The data supporting our thesis is completely available in this article.

\section{Competing interests}

All authors have no conflicts of interest or financial ties to disclose.

\section{Funding}

The paper was supported by the Natural Science Foundation of Hunan Province [grant number 2020JJ5856]. The funder, Weizheng $\mathrm{Li}$, analyzed the data concerning the treatment of the patients regarded and wrote the manuscript.

\section{Authors' contributions}

WL and KK analyzed the data concerning the treatment of the patients regarded and wrote the manuscript. SZ and $\mathrm{LZ}$ treated the patients regarded, read and approved the manuscript. PL, GW and BC contributed to the data collection and revised the manuscript critically and provided comments on the structure and grammar of the article. All authors have read and approved the manuscript.

\section{Acknowledgements}

Not applicable.

\section{References}

1. Seidell JC, Halberstadt J. The global burden of obesity and the challenges of prevention. Ann Nutr Metab. 2015 Jun 2;66(2):7-12.

2. Schauer PR, Bhatt DL, Kirwan JP, Wolski K, Aminian A, Brethauer SA, et al. Bariatric surgery versus intensive medical therapy for diabetes - 5-year outcomes. N Engl J Med. 2017 Feb 16;376(7):641-51.

3. Colquitt JL, Pickett K, Loveman E, Frampton GK. Surgery for weight loss in adults. Cochrane Database Syst Rev. 2014 Aug 8;8(8):CD003641.

4. Angrisani L, Santonicola A, lovino P, Vitiello A, Higa K, Himpens J, et al. IFSO worldwide survey 2016: primary, endoluminal, and revisional procedures. Obes Surg. 2018 Aug 18;28(12):3783-94.

5. Welbourn R, Hollyman M, Kinsman R, Dixon J, Liem R, Ottosson J, et al. Bariatric surgery worldwide: baseline demographic description and one-year outcomes from the fourth IFSO global registry report 2018. Obes Surg. 2019 Nov 12;29(3):782-95. 
6. Cadière MDPDG-B, Himpens MDJ, Germay O, Izizaw R, Degueldre MDM, Vandromme MDJ, et al. Feasibility of robotic laparoscopic surgery: 146 cases. World J Surg Oncol. 2001 Nov;25(11):1467-77.

7. Alibhai MH, Shah SK, Walker PA, Wilson EB. A review of the role of robotics in bariatric surgery. J Surg Oncol. 2015 May 7;112(3):279-83.

8. Fazl Alizadeh R, Li S, Inaba CS, Dinicu Al, Hinojosa MW, Smith BR, et al. Robotic versus laparoscopic sleeve gastrectomy: a MBSAQIP analysis. Surg Endosc. 2019 Aug 20;33(3):917-22.

9. Lundberg PW, Stoltzfus J, El Chaar M. 30-day outcomes of robot-assisted versus conventional laparoscopic sleeve gastrectomy: first analysis based on MBSAQIP. Surg Obes Relat Dis. 2019 Jan;15(1):1-7.

10. Alexandrou A, Mantonakis E, Pikoulis E, Margariti T, Dimitrokallis N, Diamantis T. Robotic sleeve gastrectomy for morbid obesity: report of a 5 year experience. Int J Med Robot. 2015 Jun 12;12(2):283-7.

11. Elli E, Gonzalez-Heredia R, Sarvepalli S, Masrur M. Laparoscopic and robotic sleeve gastrectomy: short- and long-term results. Obes Surg. 2015 Nov 23;25(6):967-74.

12. Vilallonga R, Fort JM, Caubet E, Gonzalez O, Armengol M. Robotic sleeve gastrectomy versus laparoscopic sleeve gastrectomy: a comparative study with 200 patients. Obes Surg. 2013 Jul 30;23(10):1501-7.

13. Wang W, Li J, Wang S, Su H, Jiang X. System design and animal experiment study of a novel minimally invasive surgical robot. Int J Med Robot. 2015 Apr 23;12(1):73-84.

14. Yi B, Wang G, Li J, Jiang J, Son Z, Su H, et al. Domestically produced Chinese minimally invasive surgical robot system "Micro Hand S" is applied to clinical surgery preliminarily in China. Surg Endosc. 2017 May 18;31(1):487-93.

15. Yi B, Wang G, Li J, Jiang J, Son Z, Su H, et al. The first clinical use of domestically produced Chinese minimally invasive surgical robot system “Micro Hand S". Surg Endosc. 2015 Aug 21;30(6):2649-55.

16. Guthart GS, Salisbury JK. The intuitive/sup TM/ telesurgery system: overview and application. Proceedings 2000 ICRA. Millennium Conference. IEEE International Conference on Robotics and Automation. Symposia Proceedings (Cat. No.00CH37065); 2001 Apr 24-28; San Francisco, CA, USA, USA: IEEE; 2000. p. 618-21.

17. Wang X, Wang S, Li J, Zhang G, He C. Easy grasp: a novel hybrid-driven manual medical instrument for laparoscopic surgery. Proc Inst Mech Eng C J Mech Eng Sci. 2012 Mar 15;226(12):2990-3001.

18. Li J, Wang S, Wang X, He C. Optimization of a novel mechanism for a minimally invasive surgery robot. Int J Med Robot. 2010 Mar;6(1):83-90.

19. Del Genio G, Tolone S, Gambardella C, Brusciano L, Volpe ML, Gualtieri G, et al. Sleeve Gastrectomy and Anterior Fundoplication (D-SLEEVE) Prevents Gastroesophageal Reflux in Symptomatic GERD. Obes Surg. 2020 May;30(5):1642-52.

20. Pizza F, Lucido FS, D'Antonio D, Tolone S, Gambardella C, Dell'Isola C, et al. Biliopancreatic Limb Length in One Anastomosis Gastric Bypass: Which Is the Best? Obes Surg. 2020 Oct;30(10):3685-94.

21. Wang G, Zhu L, Li W, Yang X, Li P, Zhu S. Can low BMI Chinese patients with type 2 diabetes benefit from laparoscopic Roux-en-Y gastric bypass surgery? Surg Obes Relat Dis. 2016 Dec;12(10):1890-5.

22. Freschi C, Ferrari V, Melfi F, Ferrari M, Mosca F, Cuschieri A. Technical review of the da Vinci surgical telemanipulator. Int J Med Robot. 2013 Nov 20;9(4):396-406.

23. Sang H, Wang S, Li J, He C, Zhang L, Wang X. Control design and implementation of a novel master-slave surgery robot system, MicroHand A. Int J Med Robot. 2011 Sep;7(3):334-47.

24. Vilallonga R, Fort JM, Gonzalez O, Caubet E, Boleko A, Neff KJ, et al. The initial learning curve for robot-assisted sleeve gastrectomy: a surgeon's experience while introducing the robotic technology in a bariatric surgery

Page 10/16 
department. Minim Invasive Surg. 2012 Sep 17;2012:347131.

25. Bhatia P, Bindal V, Singh R, Gonzalez-Heredia R, Kalhan S, Khetan M, et al. Robot-assisted sleeve gastrectomy in morbidly obese versus super obese patients. JSLS. 2014 Jul-Sep;18(3):e2014.00099.

\section{Figures}

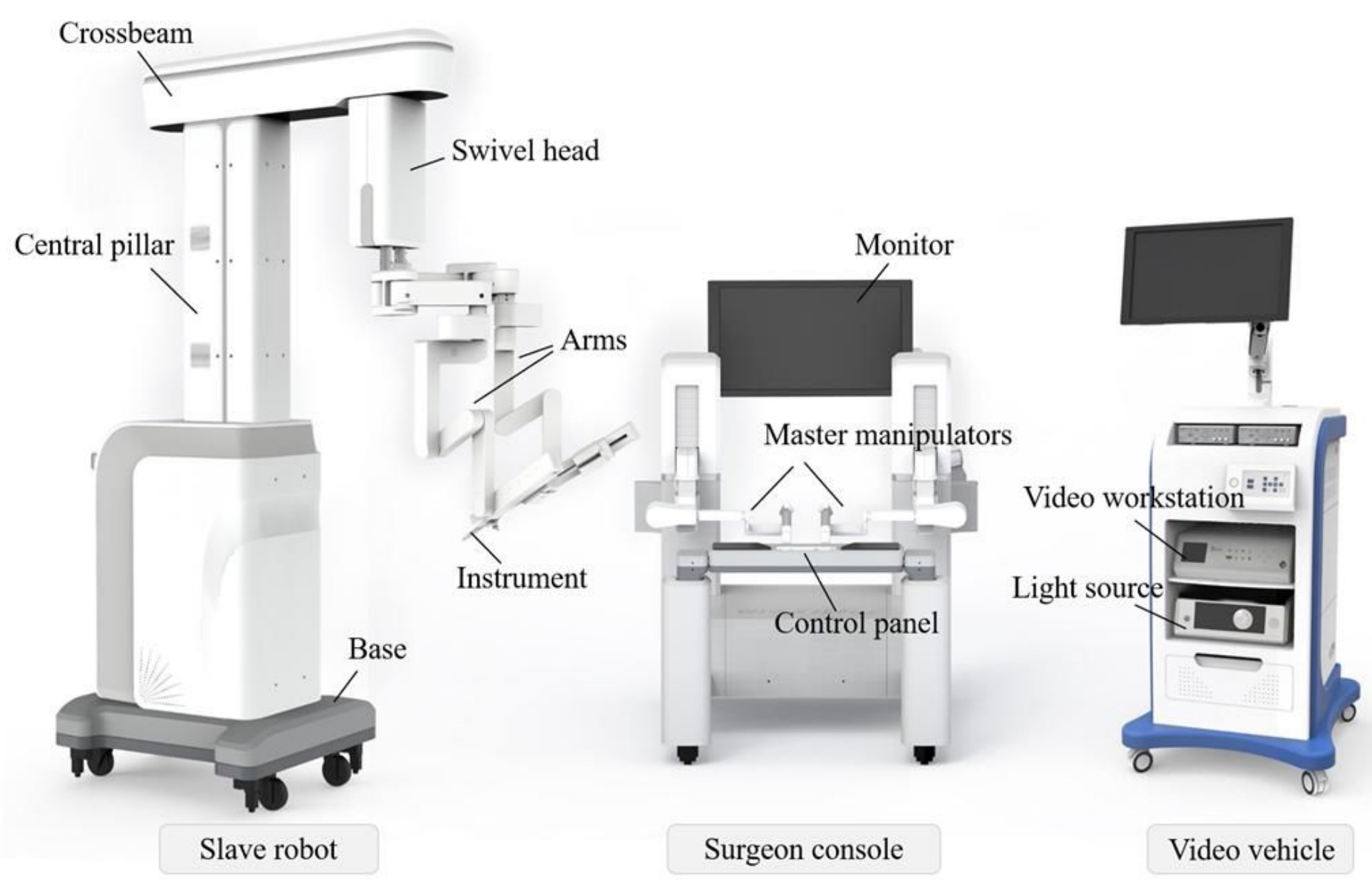

\section{Figure 1}

The structure of MicroHand S $\nabla$ robot. The system consists of a surgeon console (middle), a slave robot (left) and a video vehicle (right). An external monitor, two master manipulators and a control panel are integrated into the surgeon console. The slave robot is designed for a hoisting beam structure, which is composed of a base, a central pillar, a crossbeam, a swivel head, two arms and multi-DoF instruments. The video vehicle consists of a video workstation and light source. The central pillar is configured in the base, which can rise and fall in the vertical direction. The crossbeam is installed on the central pillar, which can translate along the horizontal axis of the pillar. A swivel head is mounted on the other end of the crossbeam. The swivel head can rotate around the vertical axis in two directions with a range of motion of $\left[-90^{\circ}, 90^{\circ}\right]$. 


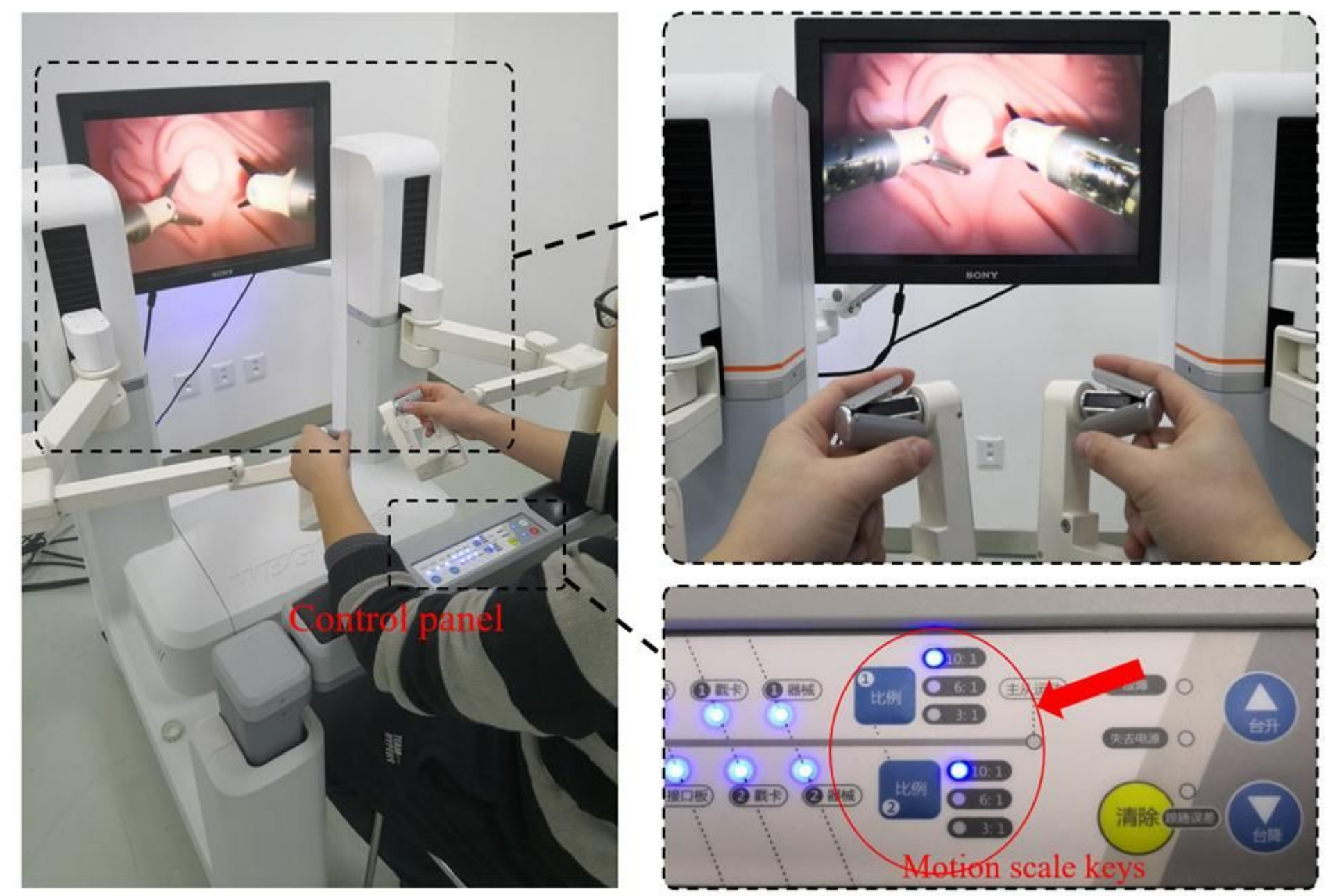

Figure 2

The structure of MicroHand S $\nabla$ robot. The system consists of a surgeon console (middle), a slave robot (left) and a video vehicle (right). An external monitor, two master manipulators and a control panel are integrated into the surgeon console. The slave robot is designed for a hoisting beam structure, which is composed of a base, a central pillar, a crossbeam, a swivel head, two arms and multi-DoF instruments. The video vehicle consists of a video workstation and light source. The central pillar is configured in the base, which can rise and fall in the vertical direction. The crossbeam is installed on the central pillar, which can translate along the horizontal axis of the pillar. A swivel head is mounted on the other end of the crossbeam. The swivel head can rotate around the vertical axis in two directions with a range of motion of $\left[-90^{\circ}, 90^{\circ}\right]$. 

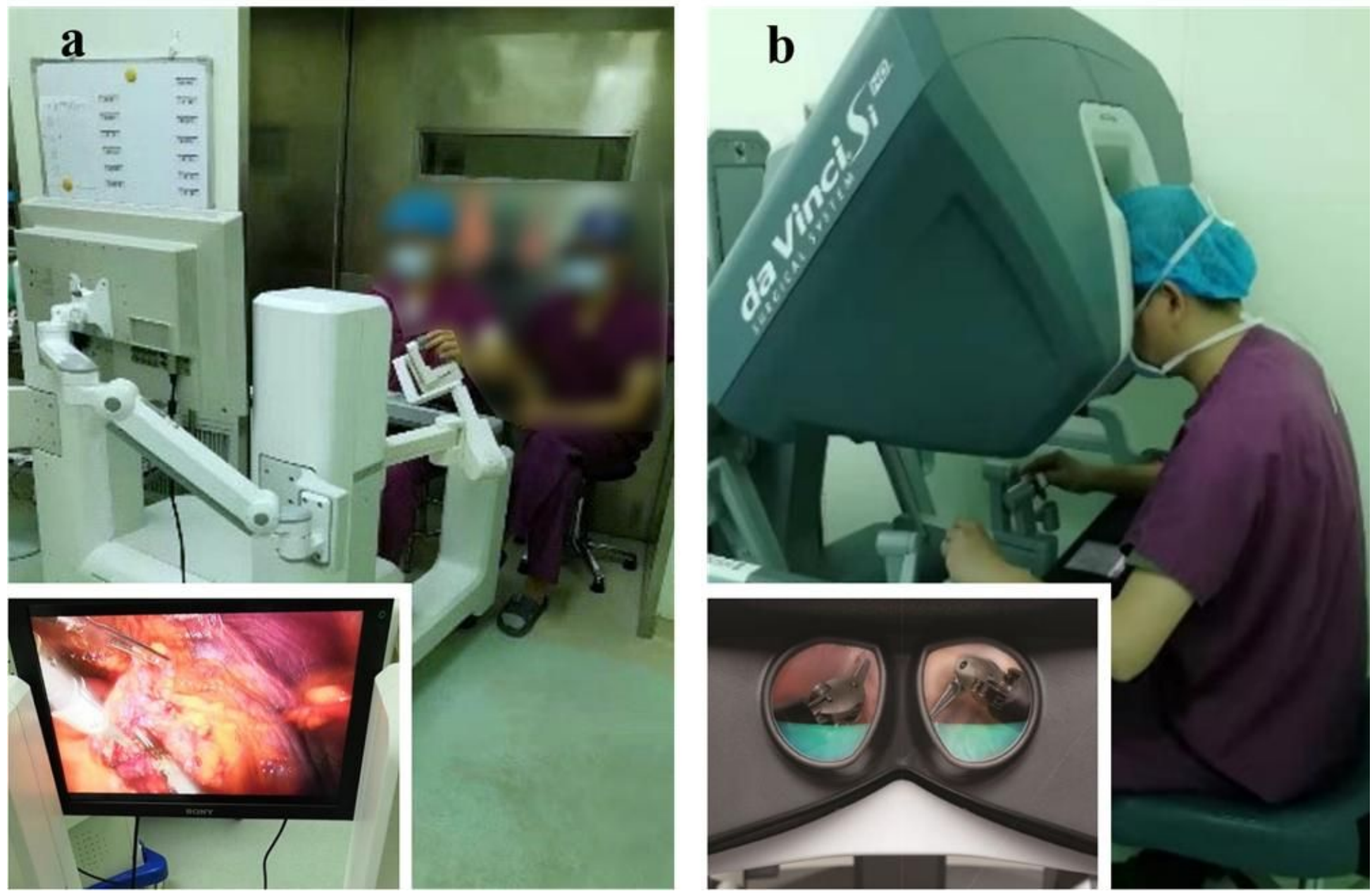

Figure 3

Fig. 3a The MicroHand S $\otimes$ system with an open-field viewer. Fig. 3b The da Vinci system with a closed image viewer. 

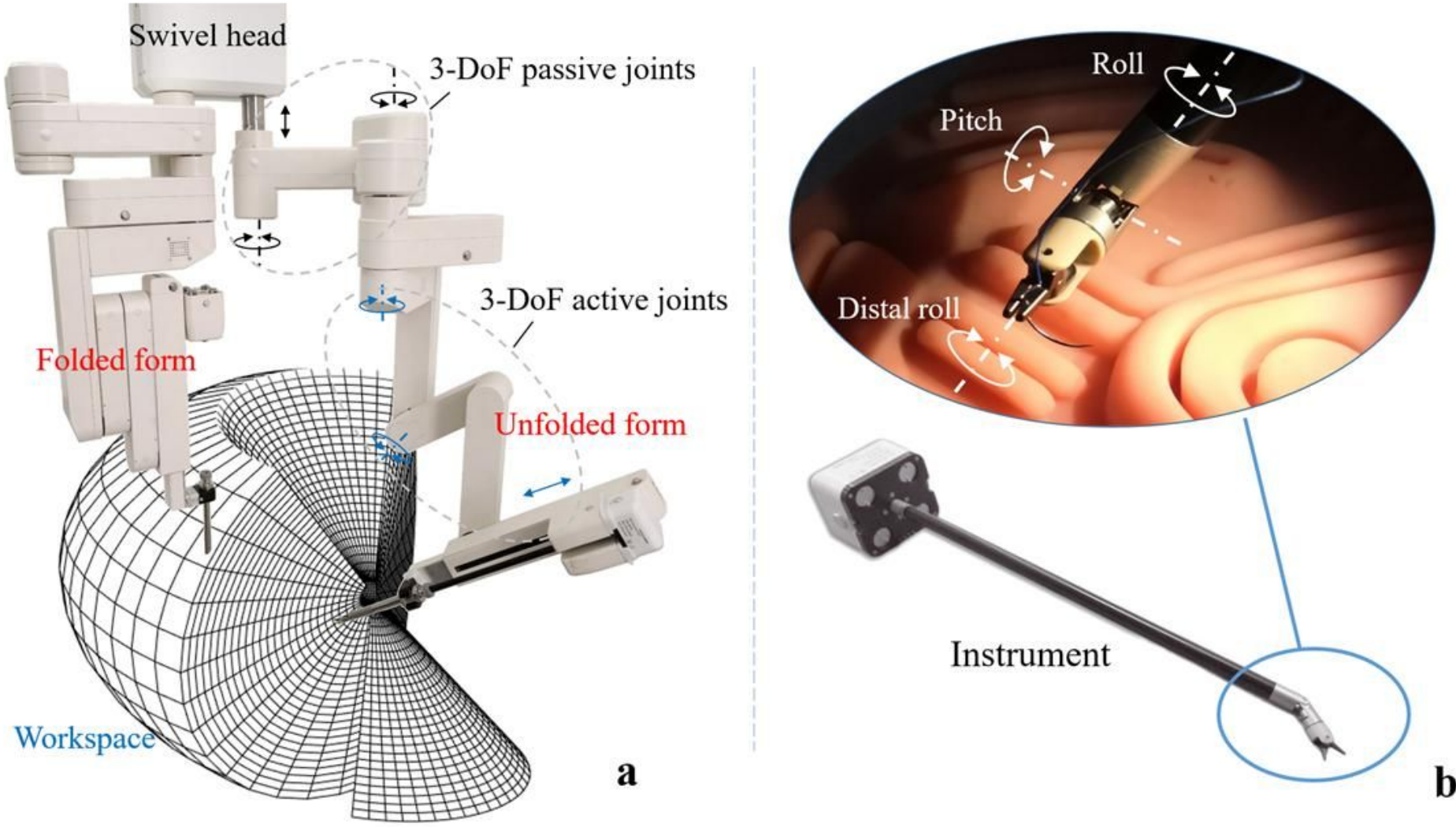

\section{Figure 4}

a The introduction of a robotic arm to MicroHand S》. The robotic arm has three active joints and three passive joints in each slave manipulator. The compact size and light weight of the operating arm are accomplished by adopting the fold-unfold structure design, which can provide a large-scale moving area. The two arms can be located in the same plane after their joints collapse tightly. The instrument can move $180^{\circ}$ in the left-to-right direction and $180^{\circ}$ in the foot-to-head direction to reach the full extent of the abdomen. The design of the active slave manipulator combined with the central pillar, crossbeam, and long-range swivel head structure can fulfil the surgical workspace of its instruments. b The multi-DoF instruments. The instrument can realize roll, pitch, and distal roll motions controlled by electrical signals transmitted from the position, orientation and grip motion of the master manipulators. 


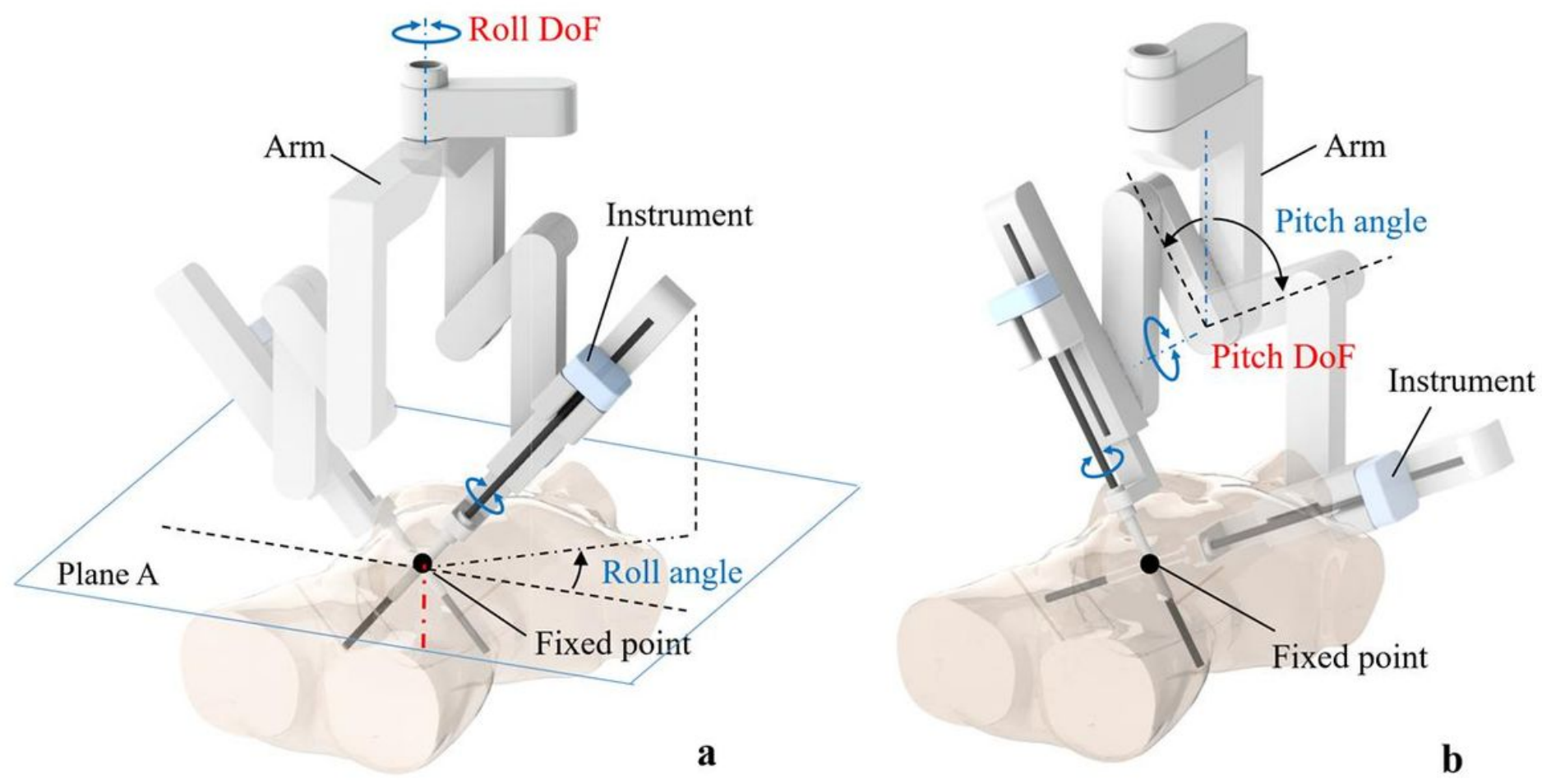

Figure 5

5a The schematic diagram of fixed point and roll angle. $b$ The schematic diagram of the fixed point and pitch angle. The fixed point acts as a fulcrum rather than the incision in the abdomen. The instrument moves around the fixed point in a roll-pitch-roll way. The course of the surgical procedure can be regarded as the combination of roll angle and pitch angle in the tridimensional workspace of the mechanism.
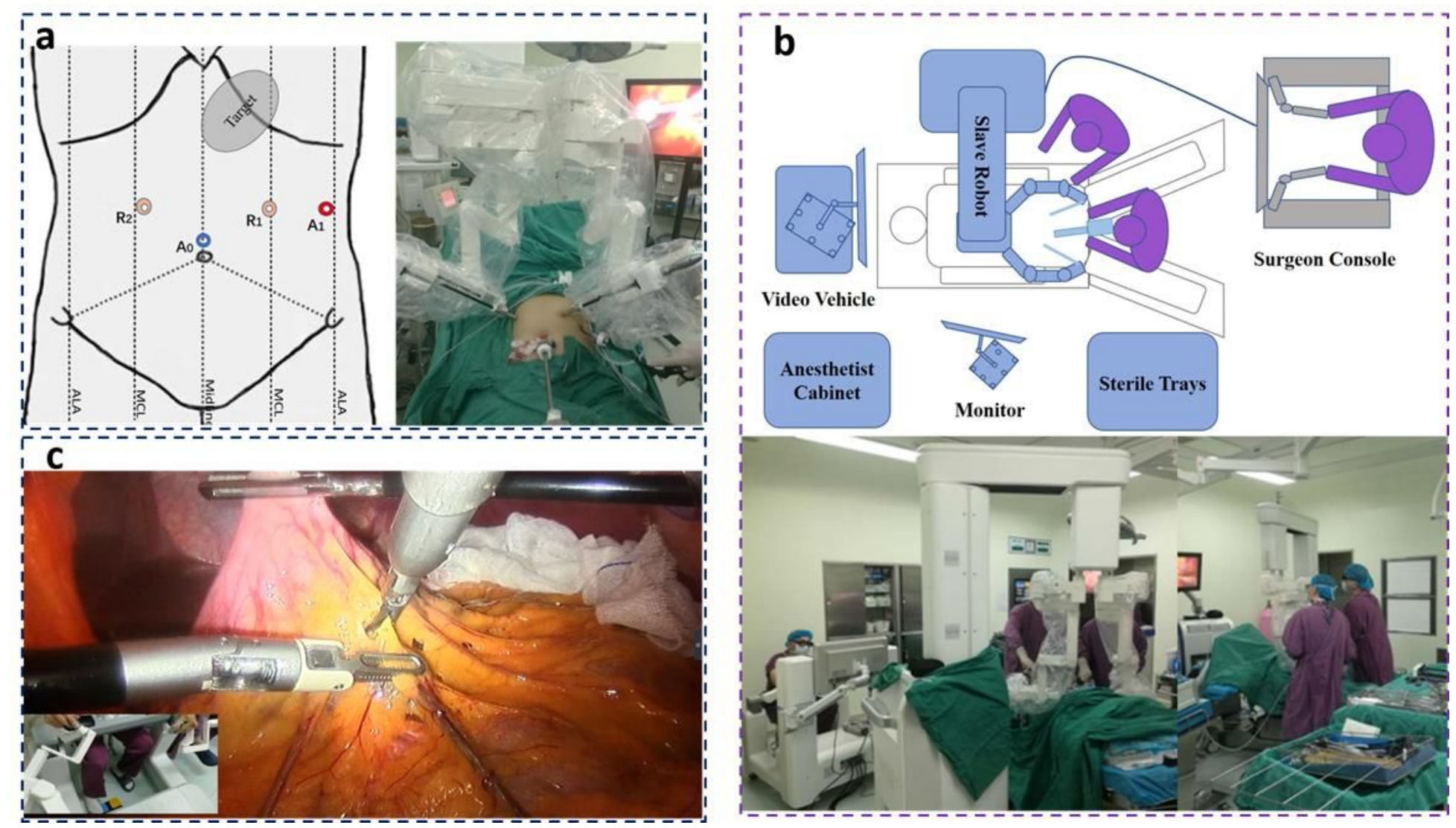


\section{Figure 6}

6a The schematic diagram and real image of port placement, showing the four incision points (R1 and R2 were used for robotic instruments, A0 was used for the laparoscope, and the last A1 was the working channel of a manual instrument, which was used to assist the robot in carrying out the surgery). b The schematic diagram and scene of

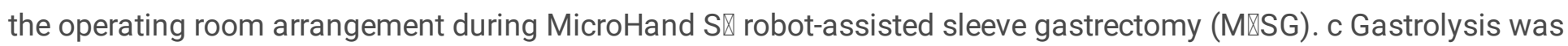
performed using a MicroHand ultrasonic scalpel.

\section{Supplementary Files}

This is a list of supplementary files associated with this preprint. Click to download.

- DR1NCFN9DD9D8468071100A001DA.pdf 